\title{
Les études de cas : un essai de synthèse
}

\author{
Paul Prévost, Ph. D. et Mario Roy, Ph. D. \\ Université de Sherbrooke
}

\section{L'ÉTUDE DE CAS}

Une étude de cas est une investigation empirique qui examine un phénomène contemporain en contexte réel lorsque les frontières entre phénomène et contexte ne sont pas clairement évidentes et pour laquelle de multiples sources d'évidences sont utilisées.

L'étude de cas est probablement le type de recherche le plus difficile à distinguer en ce moment. Selon certains auteurs, elle sera un objet d'étude particulier ${ }^{1}$, pour d'autres, elle sera une méthodologie de recherche à part entière ${ }^{1}$. Ici, elle sera considérée comme une approche de recherche plutôt que comme une méthodologie particulière. À ce titre, elle traverse l'ensemble des méthodologies ne serait-ce que parce que la notion même de «cas » se retrouve dans de nombreux domaines de la science et dans de nombreuses approches méthodologiques ${ }^{2}$. Selon $\mathrm{Yin}^{3}$, «Une étude de cas est une investigation empirique qui examine un phénomène contemporain en contexte réel lorsque les frontières entre phénomène et contexte ne sont pas clairement évidentes et pour laquelle de multiples sources d'évidences sont utilisées. » (1994 : 17)

Cette définition est probablement l'une des plus souvent reprise dans la littérature sur le domaine. Un cas peut donc être une situation, un individu, un groupe, une organisation, une décision, etc. C'est un système qui possède des frontières et chaque cas est inscrit dans un contexte essentiel à sa compréhension. Le contexte d'une étude de cas peut être conceptualisé de façon très large: enjeux historiques, sociaux, politiques ou encore de façon plus étroite : une entreprise, un service, une localisation spécifique, une famille, une période de temps, etc.
L'étude de cas unique sera privilégiée pour tester une théorie, étudier un phénomène représentant un caractère extrême ou unique ou encore révéler un phénomène qui, sans être rare, n'était pas encore disponible à la communauté scientifique.

L'étude de cas est appropriée lorsque se pose une question du type «comment» ou «pourquoi » à propos d'un ensemble contemporain d'événements, sur lesquels le chercheur n'a que peu ou pas de contrôle. Les études de cas pourront selon le besoin inclure un cas unique, plusieurs cas ou des cas imbriqués. L'étude de cas unique sera privilégiée pour tester une théorie, étudier un phénomène représentant un caractère extrême ou unique ou encore révéler un phénomène qui, sans être rare, n'était pas encore disponible à la communauté scientifique ${ }^{4}$. Elles pourront aussi chercher à mettre en évidence des conclusions spécifiques. Le cas à l'étude pourra être choisi pour ses particularités intrinsèques et on cherchera alors à le décrire ou à l'expliquer en profondeur, dans toutes ses dimensions, «pour lui-même ». Ou encore, il pourra être choisi dans une perspective plus instrumentale parce qu'il permet une nouvelle compréhension d'un phénomène donné ou le raffinement d'une théorie émergente. L'étude sera alors polarisée par une question théorique générale. Pour leur part, les études de cas multiples seront particulièrement pertinentes si on désire dégager des conclusions « générales », générer une théorie, peaufiner une théorie ou encore tester une théorie ${ }^{5}$.

Les études de cas multiples seront particulièrement pertinentes si on désire dégager des conclusions " générales », générer une théorie, peaufiner une théorie ou encore tester une théorie. 
Si une étude de cas peut être globale ou holistique, elle peut contenir aussi des sousdivisions où l'attention est concentrée de façon particulière, ce sont des cas imbriqués. Les cas imbriqués sont des enjeux spécifiques ou des aspects particuliers d'un cas à l'étude. Ce sont des sous-unités d'analyse. Aux endroits jugés significatifs, ils permettent d'ajouter plus de profondeur à la perspective globale de l'étude de cas. C'est une stratégie intéressante lorsque l'étude de cas est conduite à un niveau d'abstraction trop élevé pour traiter de certains aspects plus pointus.

Les unités d'analyse dans une étude de cas sont les unités d'information de base retenues. Elles doivent être choisies en fonction de leur degré de pertinence vis-à-vis de la question centrale de la recherche. Elles spécifient les situations étudiées. Par exemple, dans une étude de cas sur
Visa Desjardins, les unités d'analyse sont l'environnement (social, économique, technologique, législatif et industriel), l'organisation (structure, culture, technologie et processus) et les individus (dirigeants et cadres) ${ }^{6}$.

Dans l'étude d'un cas, l'unité d'analyse peut être appréhendée par rapport à l'ensemble étudié, mais aussi par rapport à des sous-ensembles jugés significatifs. On parlera alors de niveau d'analyse, tel que schématisé à la figure 1 .

À titre d'illustration, un exemple est proposé. Le cas : un service de $R \& D$ (premier niveau d'analyse); l'unité d'analyse : les employés dans le service de R\&D; sous-unité d'analyse: les employés dans les projets de $\mathrm{R} \& \mathrm{D}$; le projet devient un niveau intermédiaire d'analyse ou un cas imbriqué.

Figure 1 - Types de canevas de base pour une étude de cas

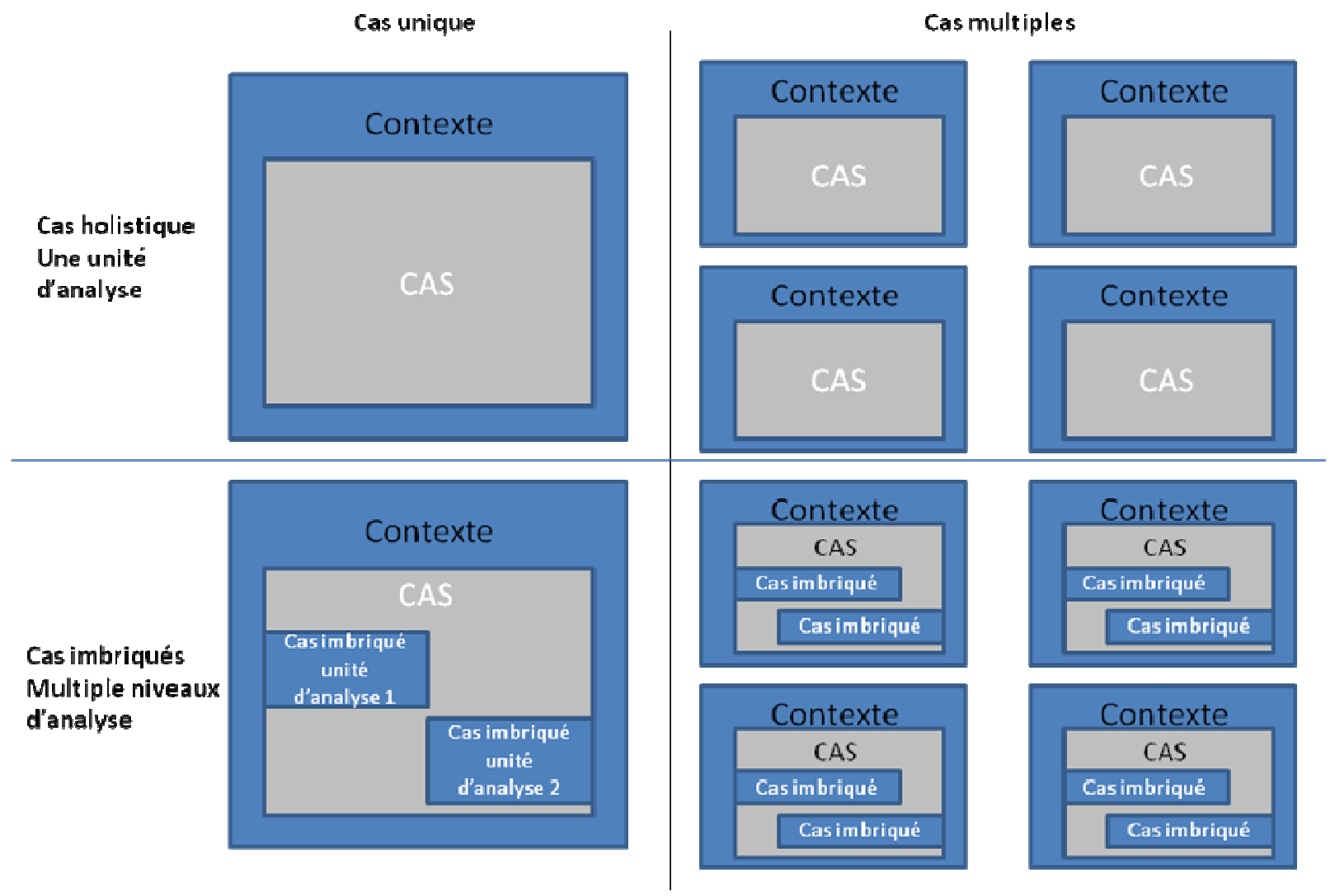


Les stratégies de recherche lorsqu'on entreprend une étude de cas pourront varier selon qu'elles sont exploratoires, descriptives ou explicatives. Elles varieront aussi évidemment selon le paradigme privilégié. Ainsi, selon le paradigme retenu, les études de cas pourront être inductives ou déductives. Dans les études de cas inductives, on voudra chercher à découvrir les concepts sous-jacents aux données recueillies sur le terrain. On se déplacera d'observations spécifiques vers le développement d'une théorie. Ce processus de traduction de données en concepts s'appelle l'abstraction. Dans une étude de cas déductive, le chercheur partira d'une théorie pour tenter de tester des hypothèses spécifiques dérivées de cette théorie. On s'interrogera alors sur le type de données à recueillir pour appréhender des concepts. On parlera alors de mesure.

\section{Les études de cas exploratoires et les études de cas descriptives tenteront d'explorer ou de décrire un phénomène contemporain en contexte.}

Les recherches dans un cas comme dans l'autre pourront être exploratoires, descriptives ou explicatives. Comme leur nom l'indique, ces études de cas exploratoires et les études de cas descriptives tenteront d'explorer ou de décrire un phénomène contemporain en contexte. Souvent perçues comme des études inférieures aux études explicatives, elles demeurent néanmoins importantes en sciences sociales et en sciences de la gestion. Même si ce n'est pas toujours le cas, il pourra y avoir dans un programme de recherche un enchainement entre ces types de recherche.

\section{Les études de cas explicatives cherchent à dégager des relations causales entre les évènements identifiés pour dégager un « pattern » capable d'expliquer des comportements ou des phénomènes.}

\section{L'ÉTUDE DE CAS UNIQUE}

L'étude de cas unique pourra être abordée de différentes manières selon les objectifs et les questions de recherche retenues. Une attention particulière a été portée dans ce texte aux études de cas exploratoires qu'elles soient déductives
Enfin, les études de cas explicatives cherchent à dégager des relations causales entre les évènements identifiés pour dégager un «pattern» capable d'expliquer des comportements ou des phénomènes. Traditionnellement, on a réservé les explications causales à la recherche quantitative. Cette vision traditionnelle repose sur la difficulté à reconnaître que les chercheurs quantitatifs et qualitatifs ne posent pas fondamentalement le même type de questions pour appréhender les relations causales dans un phénomène. Les chercheurs quantitatifs vont se demander dans quelle mesure une variance en $\mathrm{X}$ causera des effets sur $\mathrm{Y}$, appliquant ainsi une approche boîte noire à la problématique de la causalité. Alors que le chercheur qualitatif tentera d'ouvrir cette boîte pour se questionner sur les processus et les mécanismes qui relient $\mathrm{X}$ et $\mathrm{Y}$. Ce sont deux approches différentes à l'explication d'un phénomène. Sur ce point, Maxwel ${ }^{17}$ souligne que :

«Mohr (1982) call this approach to causal explanation «process theory » in distinction to variance theory. Process theory deal with events and the processes that connect them; it is bases on an analysis of the causal processes by which some events influence others. Process explanation, since it deals with specific events and processes, is less amenable to statistical approaches. It lends itself to the in-depth study of one or few cases or a relatively small number of individuals and to textual form of data that retain chronological and contextual connections between events... Both regularity/variance theory and process theory are forms of causal explanation. Process theory is not merely "descriptive" as oppose to "explanatory" variance theory; it is a different approach to explanation. » (2012: 36) 
De plus, ce sera l'occasion de réfléchir sur le rapport particulier qu'on peut y établir entre le modèle conceptuel retenu ou développé et le terrain exploré.

\subsection{L'étude de cas exploratoire}

Comme son nom l'indique, l'étude de cas exploratoire cherche simplement à explorer un phénomène. Souvent regardée comme inférieure à la recherche de type explicative, elle demeure fort intéressante sur des terrains particulièrement confus ou faire du sens avec la problématique est déjà un résultat apprécié. Une situation qui est assez fréquente en gestion. L'étude de cas exploratoire peut être une stratégie de recherche privilégiée pour étudier un phénomène peu connu ou particulièrement complexe, découvrir de nouvelles significations, peaufiner une conceptualisation novatrice ou encore transférer des conceptualisations d'un contexte théorique ou pratique à un autre. Elle peut aussi servir de stratégie partielle pour générer des hypothèses pour de nouvelles recherches ${ }^{6}$. Les questions de recherche pourront prendre les formes générales suivantes : Que se passe-t-il dans ce cas? Quelles sont les configurations, catégories ou thèmes significatifs pour les participants? Comment ces configurations sont-elles reliées?

La conceptualisation utilisée pour explorer le cas pourra avoir été élaborée en cours de route si elle est inductive ou encore, au début de l'étude si l'approche choisie est plus de type déductif. Un exemple qui vient à l'esprit à cet égard est celui de Kurt Lewin qui a adapté un modèle issu du domaine de la physique (l'analyse du champ de force) pour analyser un phénomène social. Elle sera toutefois toujours relativement flexible. De plus, qu'il apparaisse au début ou en cours de route, le modèle conceptuel issu de la conceptualisation ne prétendra pas être une représentation de la réalité. C'est plutôt un outil, un cadre de référence utile pour questionner ou explorer la réalité de façon organisée. Son rapport à la réalité n'est pas analytique, mais dialectique. S'il sert à questionner la réalité, il est en retour aussi questionné par cette réalité. Le résultat sera une meilleure compréhension de la réalité, un modèle souvent bonifié, de nouvelles pistes de recherche, etc. Dans le cadre d'une probléma- tique de changement, ce pourra aussi être un système d'apprentissage qui permettra de cheminer de façon critique d'une situation à une autre. La méthodologie des systèmes souples ${ }^{8}$ est une illustration de ce type de stratégie de recherche dont l'aller-retour entre la réalité et le modèle enclenche un processus de coconstruction pouvant même à l'occasion déboucher sur un schéma explicatif.

Les études de cas exploratoires pourront s'accommoder à l'occasion d'un échantillon de convenance, ce qui n'est pas le cas des autres types d'étude de cas qui commanderont un échantillonnage théorique.

\subsection{Les études de cas inductives}

\section{Les études de cas inductives cherchent à décrire un phénomène, à en développer une visée compréhensive et conceptualisée à partir des perceptions actions, interactions et processus des différents acteurs en présence (interprétativisme) ou à générer une nouvelle théorie à partir d'observations et de descriptions obtenues sur le terrain (constructivisme).}

Les études de cas inductives enracinent l'argumentation du chercheur dans les données recueillies sur le terrain plutôt que dans un cadre conceptuel défini a priori ${ }^{9}$. Les études de cas inductives cherchent à décrire un phénomène, à en développer une visée compréhensive et conceptualisée à partir des perceptions actions, interactions et processus des différents acteurs en présence (interprétativisme) ou à générer une nouvelle théorie à partir d'observations et de descriptions obtenues sur le terrain (constructivisme).

Si le chercheur ne fait pas appel au départ à un cadre conceptuel définitif et rigide lui prescrivant ce qu'il devrait voir, il ne fait pas pour autant abstraction de la littérature pertinente. Il pourra faire appel à un ensemble de concepts dits « sensibilisants » suggérant la direction où il faut regarder. Quoiqu'ils ne soient pas indispensables dans une recherche inductive, les concepts sensibilisants fournissent des lignes directrices 
pour aborder le terrain. Ils constituent des points de départ, des idées en arrière-plan qui informent le problème de recherche d'ensemble. Les concepts sensibilisants peuvent être efficaces pour fournir un cadre d'analyse de données empiriques et, ultimement, développer une compréhension approfondie de phénomènes sociaux. Cependant, mais qu'il est essentiel de dépasser le niveau purement descriptif pour passer à l'interprétation et à l'explication, en dégageant des thèmes ou catégories, et en construisant une théorie à partir de ceux-ci.

Les questions de recherche élaborées lors d'une étude de cas inductive par contraste avec une étude de cas déductive pourront s'inspirer des formulations suivantes :

- Qu'est-ce qui se passe ou s'est passé?
- Qui sont les acteurs significatifs?

-...les thématiques ou les problématiques émergentes les plus signifiantes?

- Quel construit théorique aide à comprendre ou expliquer ce qui se passe?

- Est-ce que ce construit est unique à ce cas?

Lors de son analyse des données, le chercheur se concentrera sur la signification des phénomènes pour en construire une compréhension en profondeur à partir des données qu'il observe, telle que présentée à la figure 2 . Le pattern, le modèle ou la théorie s'il y a lieu émergeront d'une lecture approfondie du cas à l'étude. Le modèle sera enraciné dans une réalité contextualisée. Le rapport à la réalité en est un de synthèse.

Figure 2 - L'étude de cas inductive inspirée de Creswell (1998)

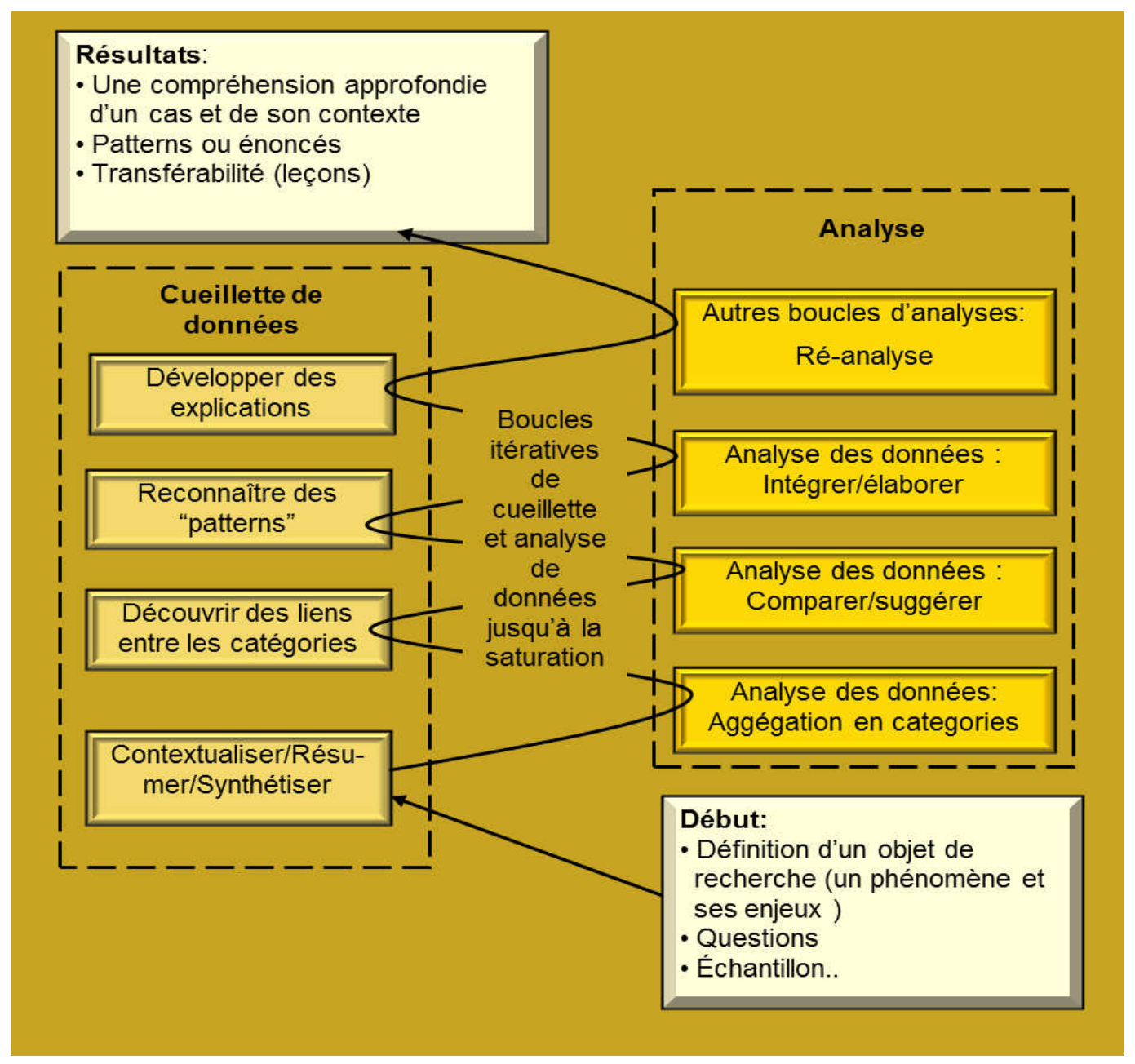


À noter ici que si la synthèse dans une étude de cas inductive peut s'apparenter à une démarche de théorisation enracinée, elle se distingue fondamentalement dans ses objectifs. La théorie enracinée vise à construire une théorie et atteindra la saturation lorsqu'aucune nouvelle donnée ne viendra enrichir de façon significative la théorie émergente. L'étude de cas pour sa part vise d'abord à comprendre en profondeur un phénomène en contexte jusqu'à saturation des données. La théorisation peut ne pas être au rendez-vous.

La démarche pourra se construire autour de plusieurs étapes telles que la description en profondeur du cas et de son contexte, l'agrégation des données en catégories, l'interprétation directe d'instances particulières puis reconstruction de façon plus significative, le dégagement de patrons ou configurations (pattern) ou d'énoncés et la transférabilité des résultats (leçons tirées du cas) ${ }^{10}$.

\subsection{Les études de cas déductives}

Les études de cas déductives s'inscrivent dans la tradition positiviste. Elles seront la plupart du temps descriptives ou explicatives et se construiront dans les deux cas autour d'une

\section{LES ÉTUDES MULTI-CAS}

Si un chercheur a le choix et les ressources, il lui sera normalement avantageux d'inclure dans son étude plusieurs cas, ne fusse que pour ne pas mettre tous ses œufs dans le même panier. Sur le plan analytique, les données seront plus riches, le chercheur pourra mettre en évidence des réplications entre les cas ou pourra confronter ses trouvailles à un cas contrasté sélectionné à cet effet pour ainsi obtenir des résultats plus robustes. Il gagnera ainsi sur le plan de la crédibilité.

\subsection{L'échantillonnage des cas}

Lorsque vient le temps de déterminer le nombre et le type de cas qui feront partie de l'échantillon, il y a de façon générale deux choix possibles, l'échantillon par choix raisonné ou échantillon théorique et l'échantillon de convenance $^{11}$. Le premier repose sur des critères théoriques tels le caractère typique ou atypique proposition théorique clairement exposée. Elles chercheront à décrire ou à expliquer les configurations (pattern) reliées au phénomène sous étude et à identifier les relations causales plausibles le structurant. Elles se questionneront sur les évènements, attitudes qui structurent le cas à l'étude ainsi que sur les forces qui interagissent pour produire le phénomène. Le rapport à la réalité, dans les études de cas déductives, sera avant tout analytique. Selon cette perspective, dans une étude de cas descriptive, la description d'une situation concrète sera effectuée à la lumière du point de vue théorique adopté. La théorie sert alors à expliciter les observations. L'étude pourra renforcer le degré de pertinence d'une théorie ou au contraire l'infirmer.

Dans une étude de cas explicative, le test de théorie se fait à l'aide d'un cas contradictoire qui vise à la falsifier en fournissant l'exemple de faits ou de phénomènes qui contredisent plusieurs des assertions théoriques. Une théorie pourra ainsi s'avérer erronée ou impropre dans une situation spécifique. L'étude peut suggérer aussi des façons d'enrichir ou corriger la théorie ${ }^{6}$.

d'un cas ou encore sa similitude ou non avec les autres cas sélectionnés. Un échantillon homogène favorisera la construction d'une théorie alors qu'un échantillon hétérogène permettra d'augmenter la robustesse d'une théorie et sa transférabilité. L'échantillon de convenance limite grandement les inférences et ne permettra que des études de cas exploratoires.

Il y a deux principes qui peuvent être utilisés pour définir la taille d'un échantillon: le principe de réplication et le principe de saturation. Selon le principe de réplication, ${ }^{4}$ chaque cas doit être soigneusement sélectionné de façon à ce qu'il prédise soit un résultat similaire, on parle alors de réplication littérale, ou qu'il prédise des résultats contrastés pour des raisons anticipées, on parle alors de réplication théorique. Si les différences sont importantes par rapport aux attentes, 2 ou 3 cas suffiront, sinon 5 ou 6 cas sont suggérés. Selon 
le principe de saturation ${ }^{12}$, la taille adéquate d'un échantillon est celle qui permet d'atteindre la saturation théorique. Ainsi, selon ce principe, chaque unité d'information supplémentaire apporte un peu moins d'information nouvelle. Ce principe s'applique aussi bien dans l'identification du nombre de cas que du nombre de répondants à l'intérieur d'un cas. On peut aussi augmenter le nombre de cas pour augmenter la transférabilité d'un modèle. On fera alors varier par exemple le contexte d'observation : localisation géographique, type d'organisation, etc.

\subsection{Les analyses multi-cas}

Les analyses multi-cas sont fondamentalement des études comparatives qui permettent de bonifier un modèle en construction ou encore de valider une proposition théorique selon que l'étude s'inscrit dans un paradigme constructiviste ou positiviste. Bien que dans la littérature les auteurs proposent plusieurs démarches, on peut les classer en deux groupes : les analyses transversales (figure 3) et les analyses par «pattern-matching » (figure 4).

Figure 3 - Analyse transversale inductive

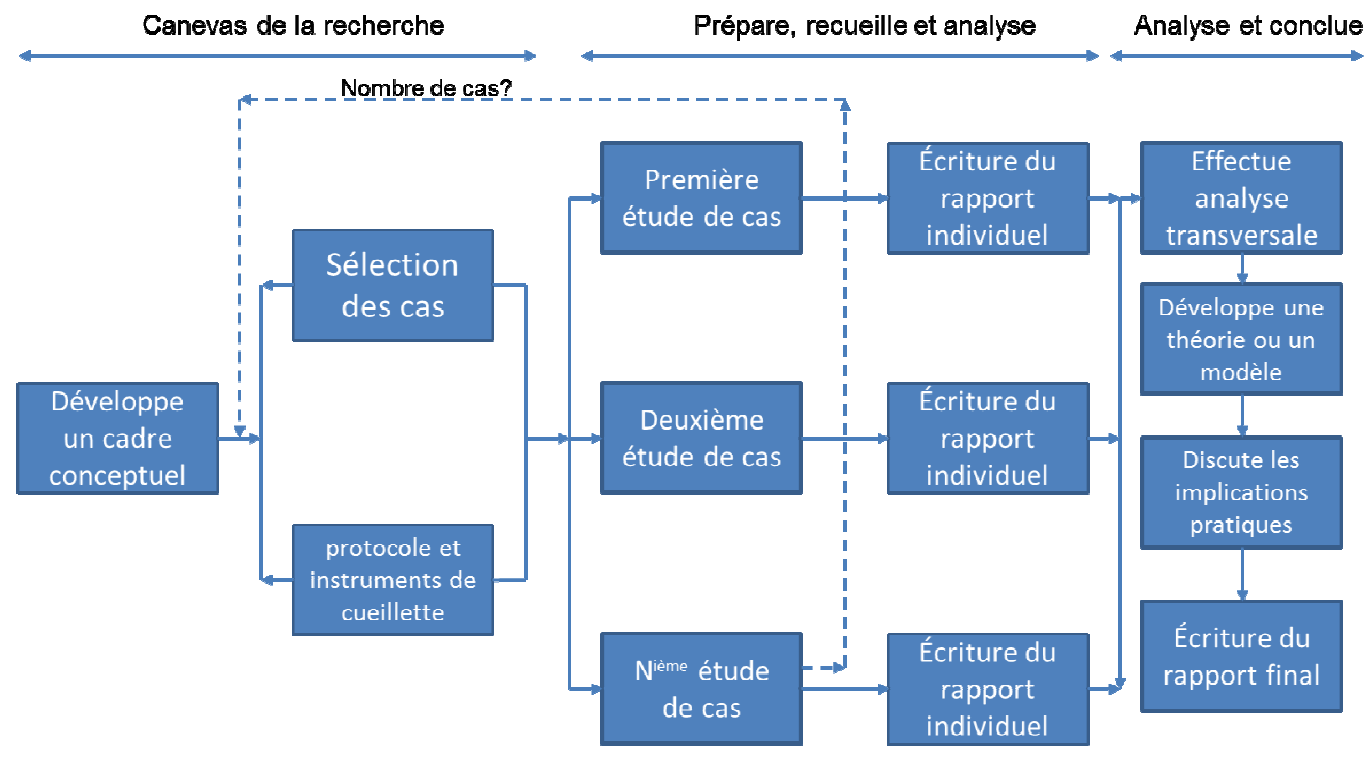

Figure 4 - Analyse transversale déductive

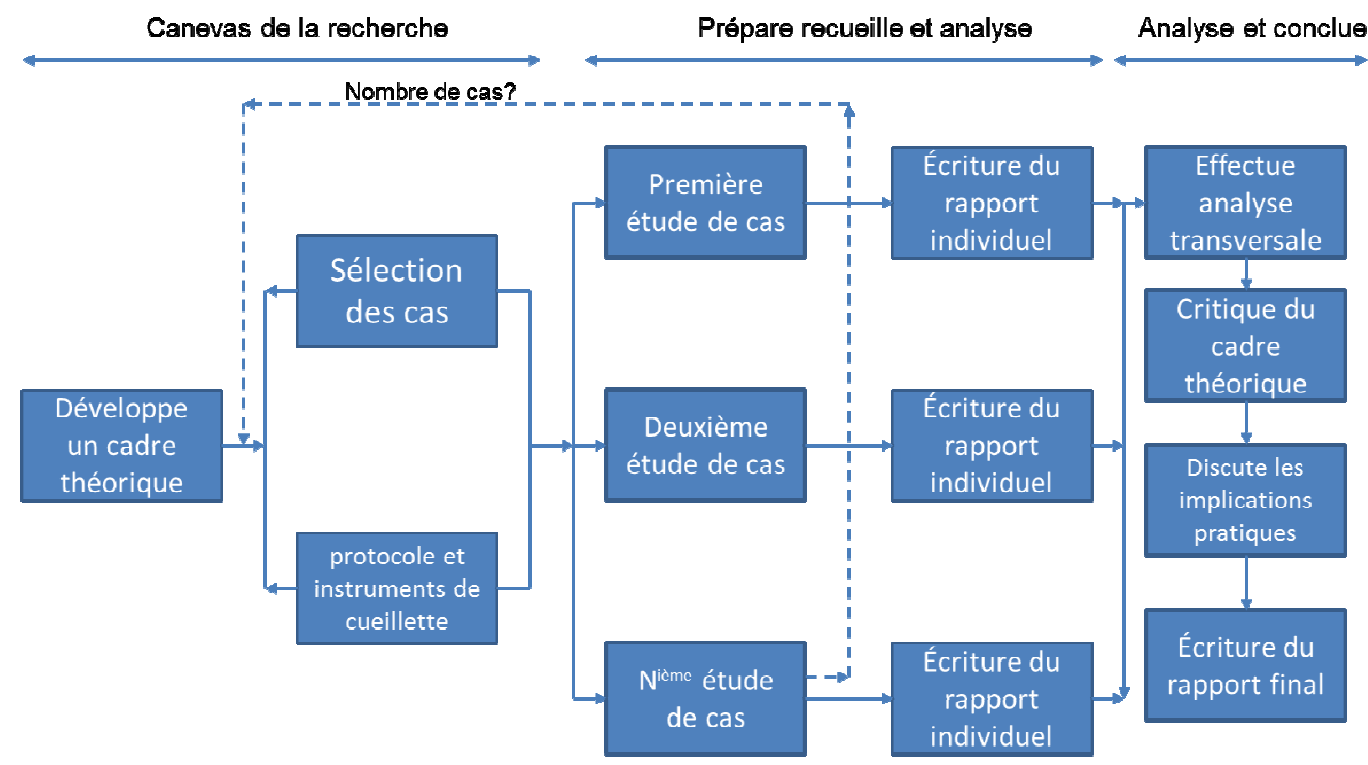


Dans les analyses transversales, si l'approche privilégiée est inductive, le chercheur se donnera d'abord un cadre conceptuel (concepts sensibilisants) pour guider initialement sa démarche puis procédera à l'étude de chaque cas sélectionné. À la fin, il superposera les résultats afin de dégager les récurrences et les divergences. C'est avec ce matériel qu'il pourra dégager la théorie ou le modèle émergent. Notez bien que les divergences ne sont pas écartées, elles doivent servir plutôt à stimuler la discussion. Est-ce que ce sont par exemple des particularités liées au contexte ou encore des trouvailles exceptionnelles qui questionnent l'intégrité du modèle émergent ou l'enrichissent?
Si l'approche de recherche est plutôt déductive, le chercheur se donnera à partir d'une revue de littérature exhaustive un cadre théorique (concepts définitifs) qui servira de référence pour analyser les cas sélectionnés et effectuer la comparaison inter-cas. Alors que dans l'approche inductive, la démarche est souple et accepte les itérations à tout moment, dans une approche déductive la démarche est rigide et vise à valider ou falsifier le modèle retenu au départ.

Les analyses multi-cas de type «patternmatching » ont été développés par plusieurs chercheurs $^{13}$ et sont schématisées dans les figures 5 et 6 .

Figure 5 - Analyse multi-cas par " pattern matching » La perspective déductive

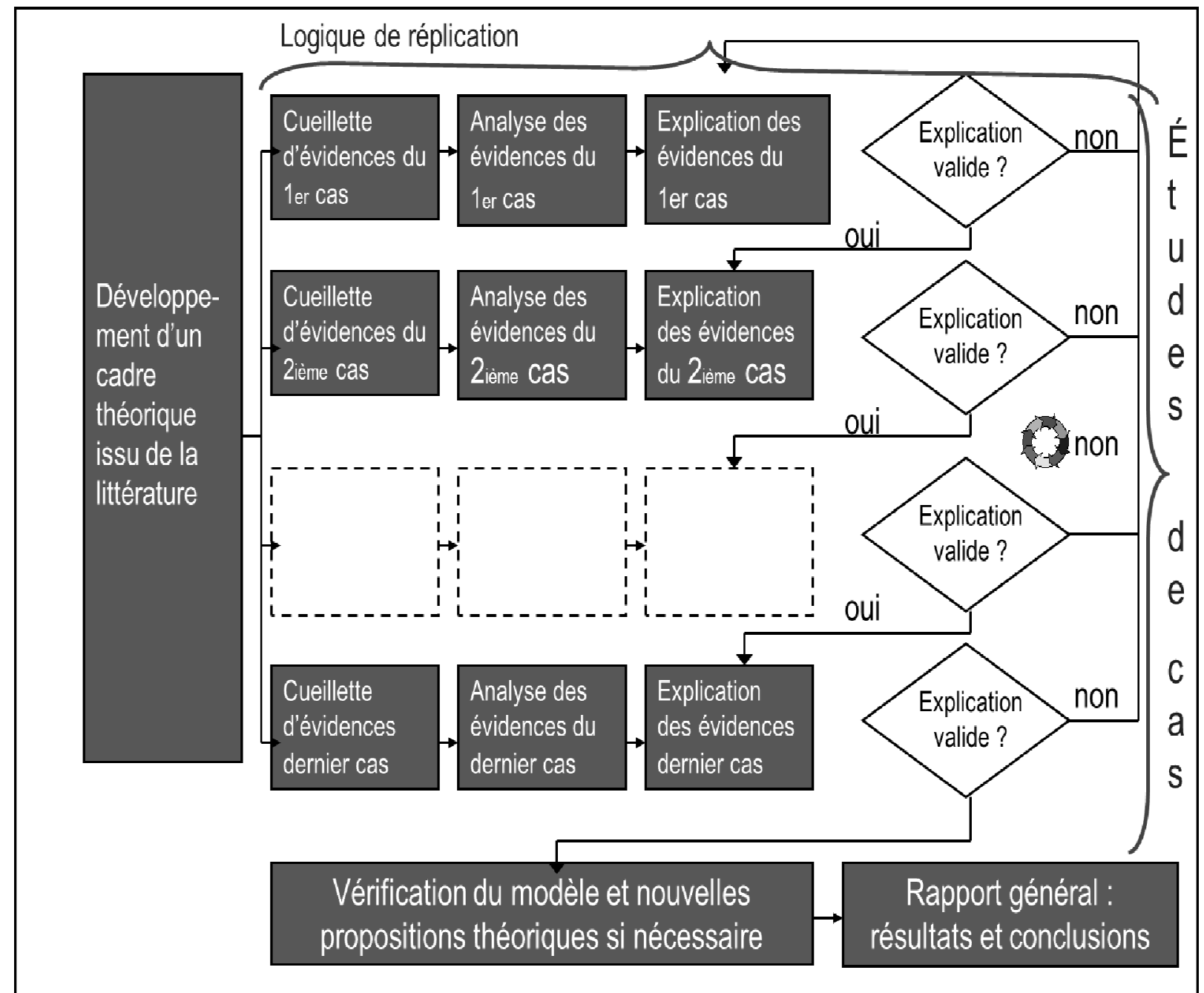


Dans ce type d'analyse déductive, le chercheur se donne au départ un pattern ou un modèle qu'il utilise comme référence pour analyser le premier cas. Les résultats de cette analyse, s'ils sont jugés valides, contribuent à l'analyse du deuxième cas et ainsi de suite jusqu'à ce que le modèle soit considéré comme étant vérifié ou falsifié. Dans une approche déductive, le pattern de départ sera issu de la littérature et restera fixe jusqu'à la fin de la recherche.

Cette stratégie d'analyse peut aussi s'utiliser lors d'une étude de cas de type inductif. Le modèle ou le «pattern » de départ ne sera pas alors issu de la littérature, mais plutôt d'une étude de cas pilote exploratoire de type inductif. Il sera par la suite appliqué à la première étude de cas et enrichi ou amendé. Ces résultats seront ensuite réinvestis successivement dans les autres cas jusqu'à saturation théorique. Notez qu'ici, à chaque application on se questionne sur la plausibilité de l'analyse et que bien que le cas contribue à l'enrichissement du «pattern»de référence, il ne remet pas en cause fondamentalement ses composantes. Dans le cas contraire, il faudrait se questionner sur la pertinence du pattern de départ et l'intérêt de continuer avec le cas suivant. En fait, on peut espérer quatre résultats d'une telle démarche :

- Le pattern de départ se maintient jusqu'à la fin;

- Le pattern évolue et se peaufine;

- Le pattern demeure intéressant, mais non concluant;

- Le pattern ne tient pas la route.

Les différentes positions présentées dans les schémas précédents ne sont pas exclusives. Elles s'inscrivent plutôt sur un continuum et, selon les problématiques de recherches abordées, le chercheur pourra se développer une stratégie d'analyse appropriée à ses questions de recherche et à son terrain. Sur ce point, il existe une variété de possibilités pour faire des analyses de type « pattern matching ».

\section{Figure 6 - Analyse multi-cas par « pattern matching » La perspective inductive}

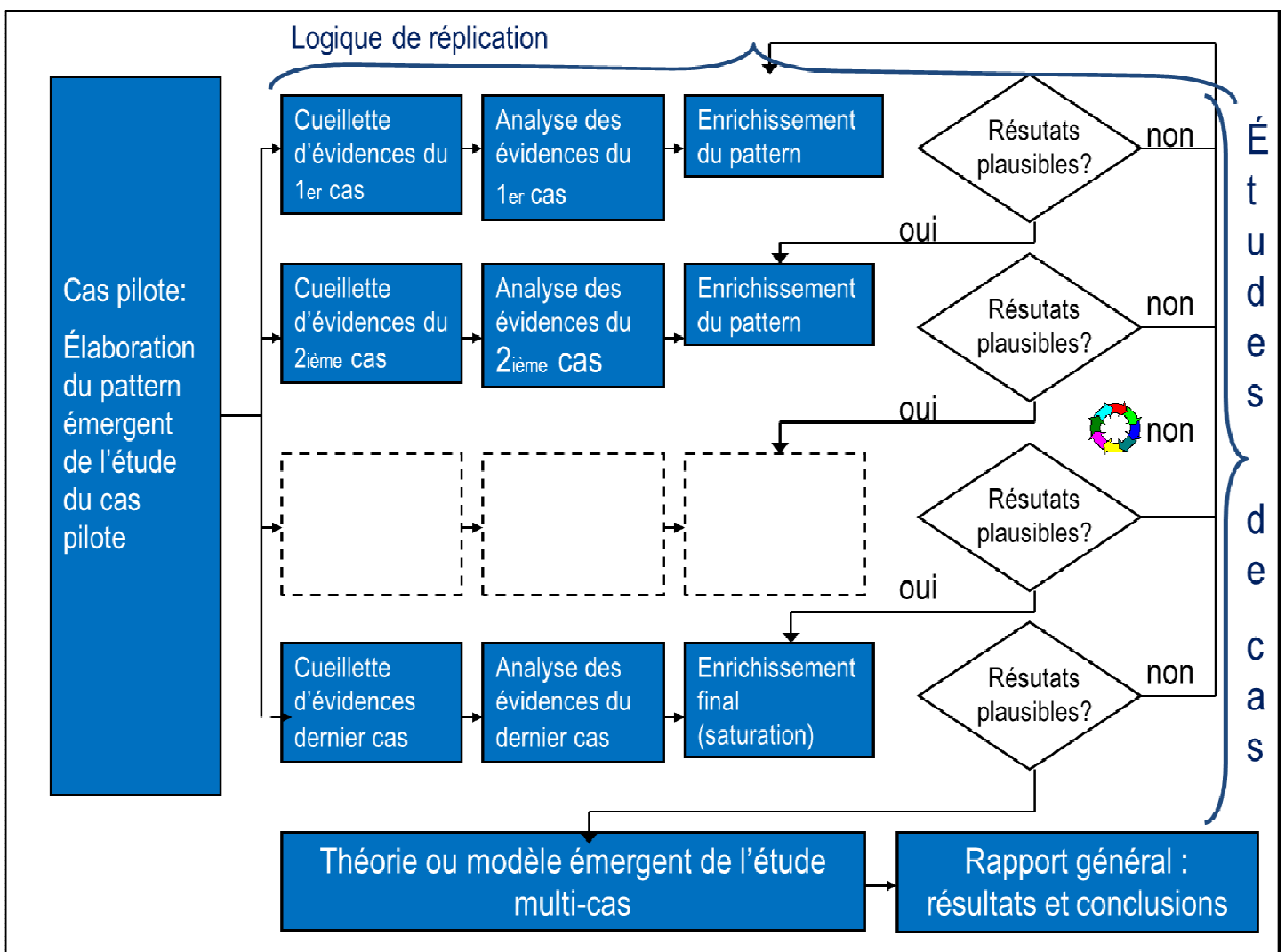




\section{L'ÉTUDE DE CAS ET LE CHANGEMENT}

En plus d'être d'une approche de recherche particulière, l'étude de cas peut permettre de diagnostiquer une situation jugée problématique, de concevoir et d'implanter des changements. Une conceptualisation a priori ou émergente peut, dans les circonstances, servir de cadre de référence pour préciser la problématique ou concevoir le ou les changements à implanter. L'étude de cas peut aussi accompagner une stratégie de recherche-action. À ce moment, une conceptualisation a priori ou une construction émergente guideront et enrichiront un ensemble

d'actions susceptibles de transformer une réalité $^{15}$. L'étude de cas peut encore s'utiliser lors d'une recherche évaluative pour apprécier les effets ou l'efficacité de quelque chose de particulier comme une innovation, une politique, un programme, un projet, une pratique ou un service. À cause du degré de confiance procuré par leur proximité avec les cas sur le terrain, les recherches qualitatives ont souvent des résultats qui ont une incidence plus importante et plus immédiate sur les changements dans les organisations $^{16}$.

\section{LA PLACE DE LA THÉORIE DANS LES ÉTUDES DE CAS}

Nous avons vu dans les sections précédentes que l'étude de cas est une approche de recherche très versatile. Elle peut adopter des formes diverses en fonction d'une variété d'objectifs auxquels elle est en mesure de répondre. La flexibilité propre à cette approche de recherche fait en sorte que la théorie, contrairement à d'autres types de recherche, peut aussi être traitée différemment d'une recherche à l'autre, telle que présentée à la figure 7 .

Figure 7 - La place de la théorie dans les études de cas

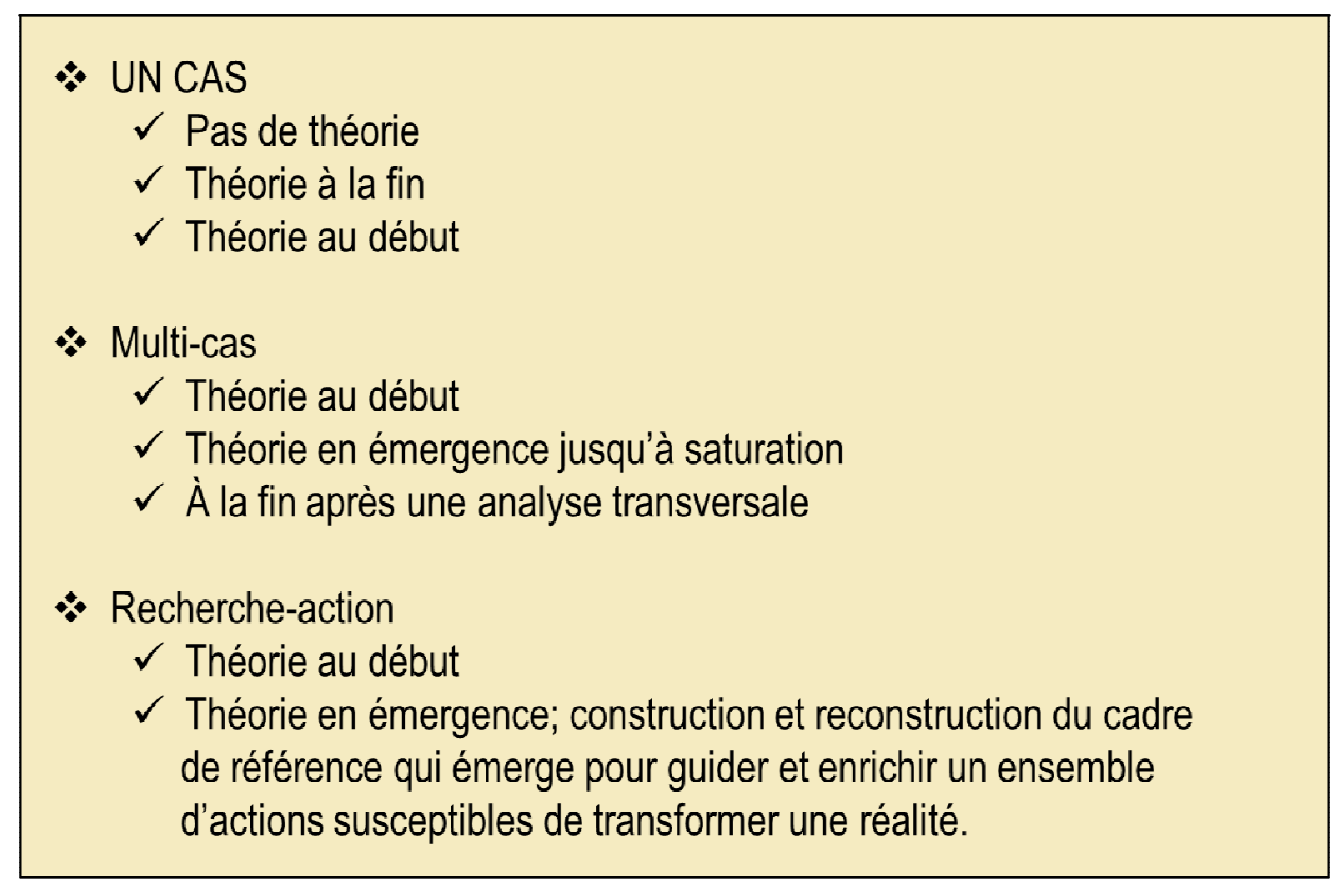

À une extrémité du continuum, un cas exploratoire inductif pourra très bien se passer de théorie au début de façon à laisser les patterns émerger en cours de route. À l'autre extrémité du continuum, un cas explicatif déductif suppose l'existence a priori d'un cadre théorique articulé que l'on voudra valider sur le terrain. L'encadré ci-dessus illustre diverses positions que la théorie peut prendre dans divers types d'études de cas. 


\section{LA CUEILLETTE DES DONNÉES}

Lorsque la recherche qualitative est employée pour réaliser une étude de cas, trois méthodes sont particulièrement populaires pour recueillir les données : l'entretien, l'observation et l'analyse documentaire.

Dans la définition présentée au début de ce texte, l'énoncé se termine en soulignant la nécessité d'utiliser de multiples sources d'évidences dans les études de cas. Lorsque la recherche qualitative est employée pour réaliser une étude de cas, trois méthodes sont particulièrement populaires pour recueillir les données : l'entretien, l'observation et l'analyse documentaire ${ }^{17}$. Elles seront brièvement présentées dans les lignes qui suivent. Notez qu'elles ne sont toutefois pas exclusives. Il existe beaucoup d'autres méthodes telles les films, les vidéos, les histoires de vie, le journal de bord, les parcours collectifs d'apprentissages, etc. Dans tous les cas, le chercheur doit se donner des outils précis et appropriés, des grilles pour consigner l'information recueillie. Ces outils seront évidemment en lien avec le cadre conceptuel et les questions de recherche.

\subsection{L'entretien}

Il y a deux types d'entretien, l'entretien individuel et l'entretien de groupe. Le premier type, l'entretien individuel est une interaction limitée et spécialisée conduite dans un but spécifique. Il apparait comme une sorte de conversation et s'apparente aux échanges verbaux informels. Mais ce n'est toutefois qu'une apparence parce qu'il est guidé par une grille d'entrevue plus ou moins élaborée selon que l'entretien soit directif ou semi-directif. C'est le chercheur qui pose les questions alors que la personne interrogée y répond. Les questions posées ne sont pas aussi précises que dans un questionnaire. Elles ouvrent sur un sujet et le chercheur encourage la personne interrogée à parler de ce qu'elle sait, il la relance, il ne cherche pas à abréger la conversation, mais à l'allonger pour en savoir plus ${ }^{18}$. Le chercheur doit montrer de l'empathie et de l'écoute envers la personne interrogée. Comme il s'agit d'un entretien qui peut évoluer plus ou moins librement, il est important, si la personne interrogée acquiesce, d'enregistrer la conversation pour entre autres s'assurer a posteriori que les sujets identifiés dans la grille ont été bien couverts.

Pour sa part, l'entretien de groupe consiste à réunir différents participants autour d'un animateur pour discuter d'un ou de plusieurs thèmes. Il fait appel à des outils comme le groupe de discussion, le remue-méninge, la technique du groupe nominal, le Delphi, etc. Les interactions entre les participants favorisent des approfondissements souvent difficiles à obtenir lors des entretiens individuels. Le succès de tels exercices repose toutefois sur les habilités de l'animateur, la qualité des participants et le respect des règles et procédures de chacune des techniques ou outils utilisés. Un accroc à ces prémisses invalidera les résultats et leur enlèvera toute pertinence pour la recherche. C'est le genre de difficulté qui empêche une utilisation plus grande de ces outils en recherche. Cependant, ce type d'entrevue n'est pas à rejeter pour autant : lorsqu'on étudie une organisation par exemple, c'est parfois le seul moyen d'accéder à l'information et il ne faut pas hésiter à y recourir.

\subsection{L'observation}

Selon Thietart ${ }^{19}$, «l'observation est un mode de collecte des données par lequel le chercheur observe de lui-même, de visu, des processus ou des comportements se déroulant dans une organisation, pendant une période de temps délimitée. L'observation constitue un mode de recueil alternatif de l'entretien dans le sens ou le chercheur peut analyser des données factuelles dont les occurrences sont certaines, plutôt que des données verbales dont l'inférence factuelle est sujette à caution. ».

L'observation pourra être participante ou non participante. Dans le cas de l'observation participante, le chercheur s'immisce dans les activités de son terrain et participe à la vie du 
groupe, de la personne ou de l'organisation qu'il veut étudier. Plus que les autres techniques de recherche qualitative, l'observation participante met l'accent sur le caractère inductif de la recherche. Dans le second cas, le chercheur prend une position externe à son objet d'étude. Il pourra observer non seulement les comportements, actions, interactions des acteurs sur le terrain, mais aussi l'environnement, le climat et un ensemble d'indicateurs non verbaux.

\section{L'une des difficultés de l'observation est la place et la relation du chercheur par rapport aux personnes observées. C'est une situation délicate qui nécessite une bonne préparation. Enfin, là comme ailleurs, le chercheur devra se donner une grille d'observation pour s'assurer de suivre le fil conducteur entre le cadre conceptuel et les questions de recherche.}

L'une des difficultés de l'observation est la place et la relation du chercheur par rapport aux personnes observées. C'est une situation délicate qui nécessite une bonne préparation. Enfin, là comme ailleurs, le chercheur devra se donner une grille d'observation pour s'assurer de suivre le fil conducteur entre le cadre conceptuel et les questions de recherche. Cette grille sera plus ou moins élaborée selon les objectifs poursuivis.

\subsection{L'analyse documentaire}

Aujourd'hui, il y a de l'information documentaire sur à peu près tous les sujets et toutes les situations. Elle constitue aussi une bonne source d'information pour n'importe quelle étude de cas. Il est donc bien important de l'inventorier et de retenir l'information pertinente à l'étude de cas en cours et aux questions de recherche qui la structurent. Toutefois, retenons que ces documents sont à peu près tous enracinés dans un contexte particulier ou biaisés à certains égards. Il faut donc les utiliser avec précaution, les croiser entre eux et les combiner avec d'autres sources de données. Enfin, ici aussi il faut planifier le travail et se donner des outils de classement et d'analyse.

\subsection{La triangulation}

Dans une étude de cas, il n'est pas recommandé de se fier à une seule source d'évidence. Les limites des méthodes utilisées, la souplesse des données qualitatives, la posture du chercheur sont toutes des sources potentielles de biais. La collecte des données à partir d'une variété de sources et de méthodes est un moyen de réduire ces problèmes, c'est ce qu'on appelle la triangulation des sources et des méthodes. Cette stratégie réduit le risque que les conclusions reflètent seulement les biais systématiques liés à une source ou à une méthode particulière de cueillette de données. De plus, elle permet d'assurer une meilleure compréhension des enjeux qui sont étudiés.

\section{Dans un exercice de triangulation, il ne faut pas seulement comparer les conclusions issues des différentes analyses, il faut trianguler aussi les données. Ainsi, les faits relatés seront supportés par plus d'une source de données.}

Dans un exercice de triangulation, il ne faut pas seulement comparer les conclusions issues des différentes analyses, il faut trianguler aussi les données. Ainsi, les faits relatés seront supportés par plus d'une source de données. La partie du bas de la figure 8 que Yin suggère pour exprimer sa pensée reconnait que certaines données ne seront pas nécessairement triangulables tout en fournissant des informations pertinentes sur l'objet d'étude".

Évidemment, l'usage de plus d'une source d'évidence sera plus coûteux à réaliser. Le chercheur devra posséder aussi des compétences techniques plus variées ou encore s'adjoindre d'autres membres à l'équipe de recherche. De plus, le chercheur devra toujours exercer son esprit critique, car les méthodes utilisées peuvent avoir les mêmes types de biais ce qui ne fera que créer un réconfort factice.

Le chercheur devra toujours exercer son esprit critique, car les méthodes utilisées peuvent avoir les mêmes types de biais ce qui ne fera que créer un réconfort factice. 
Figure 8 - Évidences convergentes

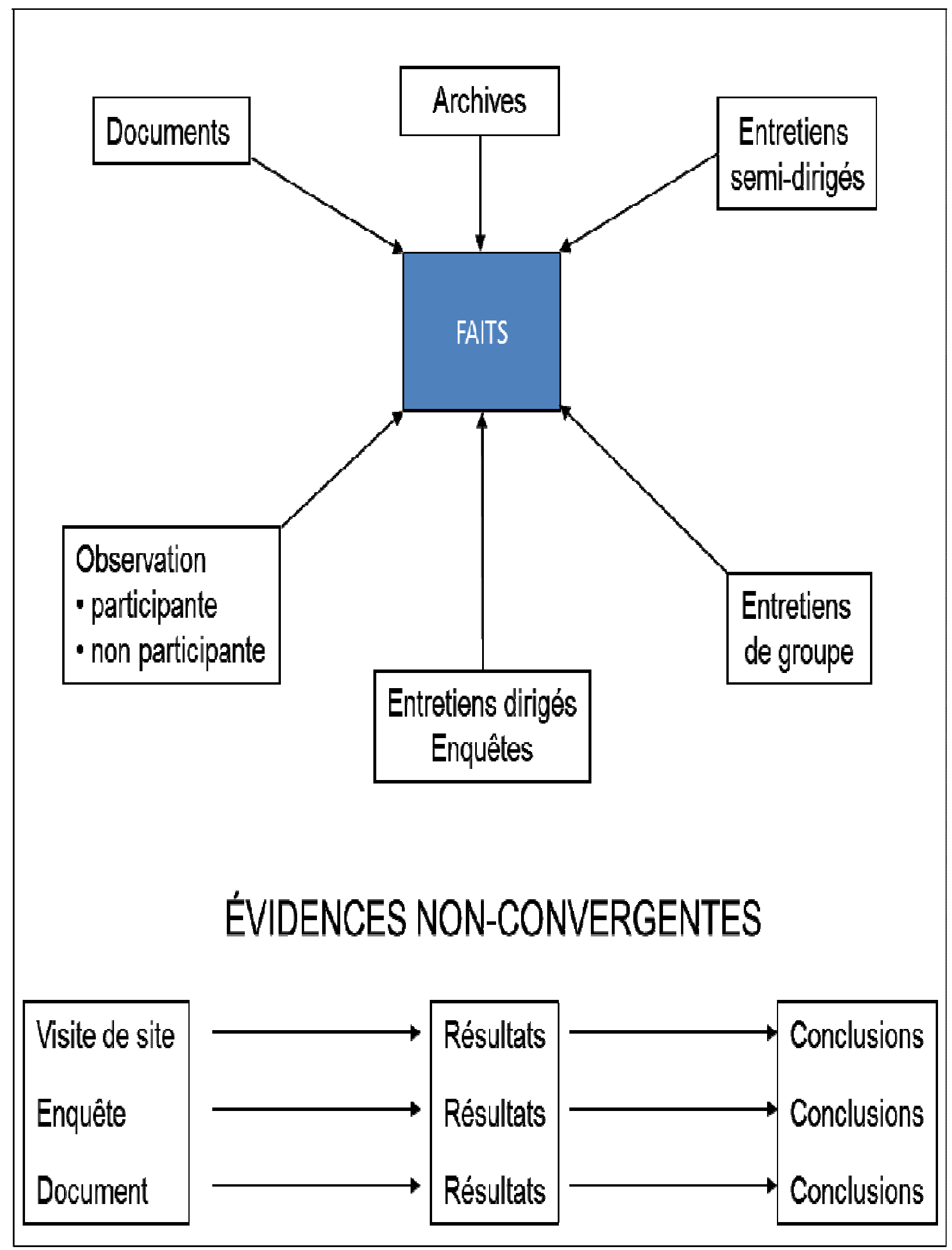




\section{LES QUALITÉS D’UNE ÉTUDE DE CAS}

\subsection{La crédibilité d'une étude de cas}

\section{Les différentes manifestations d'une absence ou d'une faiblesse de validité dans une recherche qualitative sont la quantité insuffisante d'évidences, la variété insuffisante d'évidences, l'interprétation erronée, \\ l'absence de recherche de données divergentes ou contradictoires et l'insuffisance de l'analyse de cas divergents ou contradictoires.}

La valeur scientifique de la recherche qualitative que ce soit dans les études de cas ou en lien avec d'autres stratégies de recherche est toujours objet de débat. Peu importe la position des protagonistes, la question demeure: comment fait-on pour assurer la crédibilité d'un travail qui se veut scientifique? Comment minimiser les biais? Est-il possible de généraliser des connaissances sur la base d'un échantillonnage limité? Certains chercheurs ${ }^{16}$ proposent cinq ensembles de critères pour juger la qualité d'une recherche qualitative en se référant au paradigme privilégié par le chercheur ou son domaine de recherche. D'autres proposent d'abord d'identifier les menaces à la crédibilité de la recherche puis d'élaborer des stratégies pour y faire face ${ }^{11,20}$. C'est une vision qui apparaît plus pragmatique et c'est celle que nous allons retenir. Les différentes manifestations d'une absence ou d'une faiblesse de validité dans une recherche qualitative sont la quantité insuffisante d'évidences, la variété insuffisante d'évidences, l'interprétation erronée, l'absence de recherche de données divergentes ou contradictoires et l'insuffisance de l'analyse de cas divergents ou contradictoires. Pour faire face à cette situation, certaines stratégies pourraient être envisagées :

- Formuler au début de la recherche une proposition initiale et une proposition rivale pour éviter une focalisation malsaine sur les données qui réconfortent la position du chercheur;

- Établir une chaîne d'évidence qui permet à toute personne extérieure de suivre comment les données alimentent la démarche;

- Assurer une analyse en profondeur (données riches, implication prolongée);
- Triangulation (sources, méthodes, données, chercheurs);

- Analyse de cas négatifs ou divergents;

- Faire valider la démarche par des acteurs ou des informateurs clés et des collègues.

Lorsque la recherche s'associe à une démarche d'évaluation, de changement ou de solution de problèmes, elle devra être aussi évaluée pour sa pertinence pratique. Sur ce point, il s'agit de faire valider la démarche par des acteurs ou des informateurs clés, de demander une contreexpertise et de faire l'évaluation des résultats pratiques, des effets et des impacts.

\subsection{La fiabilité de la recherche}

La fiabilité ou la fidélité d'une recherche porte sur les techniques et les instruments de collecte des données ou d'observation. Elle veut assurer la fiabilité des observations, la fiabilité des entretiens et la fiabilité des sources documentaires. Si la crédibilité signifie qu'une méthode de recherche utilisée a été capable de répondre à la question posée, la fiabilité désigne la capacité de reproduire la recherche en obtenant les mêmes résultats. La crédibilité d'une recherche suppose la fidélité, mais non l'inverse. La qualité du canevas de recherche et la précision des procédures concernant par exemple l'écriture des récits textuels condensés, la tenue du journal du chercheur, la consignation des analyses et des interprétations provisoires sont des moyens concrets d'augmenter la fiabilité d'une recherche. On peut ajouter la triangulation des méthodes et des sources et l'audit de la stratégie de cueillette de données par une personne externe. La construction d'une base de données bien organisée pour emmagasiner les données et les informations recueillies ainsi que les analyses provisoires est aussi une activité essentielle.

\subsection{La transférabilité}

La généralisation théorique n'est pas toujours aisée dans le domaine des sciences sociales et humaines. Sur ce point, Deslauriers est précis en notant: «Pourquoi toujours chercher à généraliser? Bien sûr, plus une théorie s'applique à un grand nombre de cas différents plus elle est efficace; c'est le 
critère que la science s'est fixée. Cependant la généralisation est-elle toujours possible ou même souhaitable?... La généralisation des résultats suppose un contexte stable et une sorte de déterminisme qui ne se retrouve jamais tout à fait dans la vie sociale. De sorte que la généralisation est difficile. »

Cependant, c'est un effort qui n'est pas dénué d'intérêt. Car, même si l'exercice a ses limites, les concepts générés par une étude de cas ont parfois une utilité externe qui dépasse les attentes. Les «principes de management» par exemple ont été développés par Fayol au début du siècle dernier suite à une réflexion sur sa carrière et ses pratiques de gestion. Suggérés par l'auteur comme moyens pour assurer une bonne gestion et donc le succès, ces principes, parce que les entreprises sont complexes et non homogènes, n'ont jamais en pratique garanti ce succès et n'ont jamais été par ailleurs validés statistiquement ${ }^{21}$. Ils continuent tout de même à être enseignés à tous les nouveaux étudiants en gestion parce qu'ils sont après tout ce temps encore utiles malgré leurs limites pour comprendre la gestion des entreprises.

Autre illustration, le modèle de planification stratégique FFMO (forces, faiblesses, menaces, opportunités) n'a jamais assuré les résultats escomptés, la réalité est trop complexe et trop mouvante. Néanmoins, il demeure utile pour élaborer de façon organisée les orientations d'une organisation et faire en connaissance de cause les ajustements en cours de route. Ce modèle n'est pas complètement prédictif, ni normatif, c'est un système d'apprentissage qui permet à des gestionnaires d'apprendre sur leur gestion stratégique, leur organisation et leur champ d'action et de prendre leurs décisions en conséquence.

Donc, comme le chercheur dans une étude cas est plus préoccupé par la profondeur de son étude que par l'étendue de son échantillon, la généralisation de ses résultats ne pourra pas se baser sur leur réplication statistique à d'autres échantillons comme le veut la pratique en recherche quantitative. En recherche qualitative, on parlera plutôt des leçons tirées de l'étude de cas et de leur transférabilité en tout ou en partie à d'autres cas et sous quelles conditions.

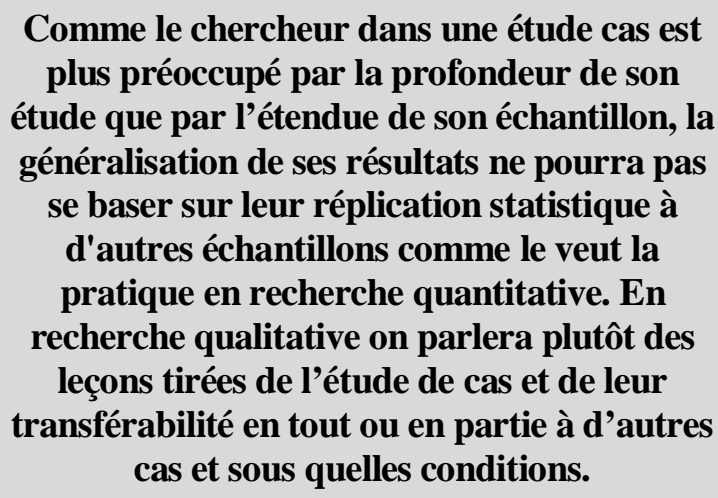

Comme le chercheur dans une étude cas est plus préoccupé par la profondeur de son étude que par l'étendue de son échantillon, la généralisation de ses résultats ne pourra pas se baser sur leur réplication statistique à d'autres échantillons comme le veut la pratique en recherche quantitative. En recherche qualitative on parlera plutôt des leçons tirées de l'étude de cas et de leur transférabilité en tout ou en partie à d'autres cas et sous quelles conditions.

On pourra ainsi améliorer le potentiel de transférabilité des résultats d'une étude de cas à un autre cas en s'assurant qu'il a été bien étudié en profondeur et contextualisé, comme l'a souligné David $^{2}$ :

«À cet égard, il faut donc, pour transférer les résultats d'une étude de cas, être capable non seulement de décrire précisément le contexte (telle entreprise, tel projet, etc.), mais aussi, et surtout, de dire de quel genre de contexte il s'agit. Le fait que ce type de résultat soit éventuellement plus conjectural que celui d'une approche quantitative ne change pas grand chose : un résultat, quel qu'il soit, n'est transférable que s'il s'accompagne d'un certain nombre de clés permettant de maîtriser un processus de transposition qui reste, en son point de départ, au moins partiellement conjectural. » (2004:14)

Le transfert des résultats analytiques ou théoriques d'un cas à d'autres cas peut permettre d'en peaufiner la conceptualisation si les cas sont relativement homogènes ou de la rendre plus robuste si les cas sont contrastés et la mettre à l'épreuve dans un autre contexte.

Enfin, une comparaison des résultats d'une étude de cas avec la littérature pertinente pourra aussi permettre d'en évaluer la contribution et de raffiner encore plus la conceptualisation, ce qui permettra d'augmenter encore son potentiel de transférabilité. 


\section{CONCLUSION}

Fondamentalement, l'étude de cas permet de comprendre en profondeur un phénomène complexe situé dans son contexte. Le chercheur pourra aussi à partir de son étude vouloir développer une théorie pour expliquer le phénomène à l'étude ou le prendre à témoin pour valider une théorie. Il pourra aussi vouloir s'appuyer sur une étude de cas pour initier un changement, l'accompagner ou l'évaluer. Chaque objectif poursuivi nécessitera une stratégie et des méthodes de cueillette et d'analyse des données appropriées. La proximité du chercheur avec les personnes interrogées nécessitera toujours un cadre éthique mutuellement accepté et des précautions particulières pour éviter la contamination des données.

Dans ce texte, il est fait exclusivement référence aux aspects qualitatifs des études de cas. En réalité, et en particulier en sciences de la gestion, les études de cas peuvent contenir aussi des données quantitatives pour enrichir la compréhension du phénomène étudié. Comme d'ailleurs une étude quantitative pourra à l'occasion faire appel à l'étude de cas qualitative pour structurer une hypothèse de recherche ou encore approfondir certains résultats.

\section{BIBLIOGRAPHIE}

${ }^{1}$ Eisenhard, K. (1989). Building Theories from Case Study Research. Academy of Management Review, Vol. 14 (4), 532-550. Robson, C. (1993). Real World Research: A Resource for Social Scientist and Practitioner Researchers. Blackwell. ${ }^{1}$ Stake R., (1995).The Art of Case Study Reseach. Thousand Oaks, CA: Sage.

${ }^{2}$ David, A., (2004). Études de cas et généralisation scientifique en sciences de gestion. École Normale Supérieure de Cachan. Saisi sur internet, décembre 2011. ${ }^{3}$ Yin, Robert K. (1994). Case study research. Design methods, ( $2^{\mathrm{e}}$ éd.). London: Sage Publications.

${ }^{4}$ Yin, Robert K. (2009). Case study research. Design methods, (4 éd.). London: Sage Publications.

${ }^{5}$ Gummesson, E. (2000). Qualitative Methods in Management Research, 2d edition. CA: Sage Publications.

${ }^{6}$ Hlady Rispal M., (2002). La méthode des cas. De Boeck Université, Bruxelles.

${ }^{7}$ Maxwell, J. A. (2012). A Realist Approach for Qualitative Research Design, Sage Publications.
Miles, M. B., Huberman, M. (2003). Analyse des données qualitatives. DeBoeck.

${ }^{8}$ Checkland, P. (1999). Systems thinking, Systems practice: Includes a 30 Years Retrospective. John Wiley \& Sons, England.

${ }^{9}$ Guba E. Lincoln Y. (1985). Naturalistic Inquiry. Beverly Hills, CA: Sage Publications.

${ }^{10}$ Creswell, J. W. (2003). Research Design: Quantitative, Qualitative, and Mixed Methods Approaches. SAGE. Thousand Oaks. USA.

${ }^{11}$ Pires, A., (1997) "Échantillonnage et recherche qualitative: essai théorique et méthodologique" publié dans un ouvrage sous la direction de Poupart, Deslauriers, Groulx, Laperrière, Mayer, Pires, La recherche qualitative. Enjeux épistémologiques et méthodologiques, pp. 113-169. Montréal: Gaëtan Morin, Éditeur, 1997, 405 pp.

${ }^{12}$ Glaser B. G, Strauss A. A. (2010). La découverte de la théorie ancrée, Armand Colin.

${ }^{13}$ Robson, C. (2011). Real World Research. Third edition, Wiley.

${ }^{14}$ Nieto, M., Pérez, W. (2000). The Development of Theories from the Analysis of the Organization: Case of Studies by the Pattern of Behavior. Management Decision, Vol. 38 (10), 723-733. Yin, R. (1997). The Abridged Version of Case Study Research, in Bickman L. y Rog D.J., Handbook of applied social research methods, Sage Publications. Yin, Robert K. (2003). Case study research. Design methods, ( ${ }^{\mathrm{e}}$ éd.). London: Sage Publications.

${ }^{15}$ Checkland, P. B., \& Holwell, S. (1998). Action research: Its nature and validity. Systemic Practice and Action Research, 11(1), 9-21. Cunningham, J. B. (1993). Action research and organizational development. Westport, CT: Praeger. Greenwood, D. J., \& Levin, M. (1998). Introduction to action research: Social research for social change. Thousand Oaks, CA: Sage.

${ }^{16}$ Guba E., Lincoln, Y. (1989). Fourth Generation Evaluation, Newbury Park, CA: Sage. Patton M., Q., (2002), Qualitative Research \& Evaluative Méthods, 3 ed., Sage. Robson, C. (2002). Real World Research: A Resource For Social Scientist And Practitioner Researchers. Second edition, Backwell.

${ }^{17}$ Lessard-Hébert, M., Goyette, G., et Boutin, G. (1997). La recherche qualitative: Fondements et pratiques, Québec, De Boeck Université.

${ }^{18}$ Deslauriers, J-P. (1991). La recherche qualitative: guide pratique, McGraw-Hill, Montréal.

${ }^{19}$ Thiétart R-A ., (1999). Méthode de recherche en management, Paris, Dunod.

${ }^{20}$ Maxwell, J. A. (2005). Qualitative Research Design: an interactive approach, Sage Publications.

${ }^{21}$ Mintzberg, H. (1973). The Nature of Managerial Work, New York, Harper \& Row. 